\title{
Lasers aplicados à frenectomia em pacientes pediátricos com anquiloglossia
}

\author{
Lasers applied to frenectomy in pediatric patients with ankyloglossia \\ Láseres aplicados à frenectomia em pacientes pediátricos com anquiloglossia
}

Recebido: 19/01/2021 | Revisado: 21/01/2021 |Aceito: 04/02/2021 | Publicado: 10/02/2021

José Edson Pavini Nunes

ORCID: https://orcid.org/0000-0002-9379-2523

Universidade Brasil, Brasil

E-mail: jpavini@hotmail.com

Ayrthon Lucena da Silva

ORCID: https://orcid.org/0000-0001-7437-0395 Universidade do Estado do Mato Grosso, Brasil

E-mail: ayrthon.mes=d@gmail.com

Guilherme Pavini Nunes

ORCID: https://orcid.org/0000- 0003-1789-8286

Universidade do Estado do Mato Grosso, Brasil

E-mail: gpavini1@ @otmail.com

Caroline Ferreira Fagundes

ORCID: https://orcid.org/0000-0002-9928-2065

Universidade do Estado do Mato Grosso, Brasil

E-mail: caroline.fagundes@unemat.br

Matheus Rodrigues de Souza

ORCID: https://orcid.org/0000-0001-5517-0777

Universidade do Estado do Mato Grosso, Brasil

E-mail: matheus.rodrigues@unemat.br

Ricardo Scarparo Navarro

ORCID: https://orcid.org/0000-0003-4699-3434

Universidade Brasil, Brasil

E-mail: ricardosnavarro@gmail.com

\begin{abstract}
Resumo
A anquiloglossia, ou língua presa, é uma condição pela qual o frênulo lingual se fixa próximo à ponta da língua e pode ser curto, tenso e grosso. A anquiloglossia foi citada como uma causa de amamentação inadequada e dor nos mamilos maternos. A frenotomia ou frenectomia pode corrigir a restrição ao movimento da língua e permitir uma amamentação mais eficaz com menos dor no mamilo materno. Tradicionalmente, a intervenção cirúrgica é realizada com bisturi, porém nos últimos anos houve um importante advento do uso do laser neste procedimento. Objetivo: realizar uma revisão sistemática sobre o uso de lasers como terapia para anquiloglossia. Métodos: revisão sistemática de estudos clínicos randomizados com pontuação $\geq 6$ na escala PEDro (Physiotherapy Evidence Database) publicados nas bases de dados PubMed, Cochrane e SciELO. Foram selecionados estudos que avaliaram o uso do laser no tratamento de crianças com anquiloglossia. Resultados: No total, foram analisados 7 estudos utilizando o laser Diodo ou $\mathrm{CO}_{2}$ como uma das técnicas de frenectomia. Em 401 pacientes submetidos à frenectomia lingual, o benefício entre a realização da técnica a laser sobre a técnica de bisturi tradicional está claramente descrito. Conclusão: A cirurgia de frenectomia lingual com lasers proporciona um tratamento mais eficiente e confortável para o paciente infantil em comparação aos métodos tradicionais de bisturi/lâmina. Porém, ainda não há consenso ou evidência para indicar o comprimento de onda laser a ser utilizado.
\end{abstract}

Palavras-chave: Frenectomia lingual; Anquiloglossia; Laser.

\begin{abstract}
Tongue-tie or ankyloglossia, is a condition whereby the lingual frenulum attaches near the tip of the tongue and may be short, tight and thick. Tongue-tie has been cited as a cause of poor breastfeeding and maternal nipple pain. Frenectomy, which is commonly performed, may correct the restriction to tongue movement and allow more effective breastfeeding with less maternal nipple pain. Traditionally, surgical intervention is performed using a scalpel, however in the last years there has been an important advent of using lasers in this procedure. Objective: carry out a systematic review on the use of lasers as therapy for ankyloglossia. Methods: systematic review of randomized clinical studies with a score $\geq 6$ on the PEDro scale (Physiotherapy Evidence Database) published in the databases including PubMed, Cochrane and SciELO. Studies that evaluated the use of laser in the treatment of children with ankyloglossia were selected. Results: In total, 7 studies using laser (Diode or $\mathrm{CO}_{2}$ ) as one of the techniques for frenectomy were analyzed. In 401 patients underwent lingual frenectomy the benefit between performing the laser technique over the traditional scalpel technique is clearly described. Conclusion: Frenectomy surgery using lasers
\end{abstract}


provides a more efficient and comfortable treatment for the child patient compared to traditional scalpel/blade methods. However, there is no consensus or evidence to indicate the type of laser to be used.

Keywords: Ankyloglossia; Lingual frenectomy; Laser applications.

\begin{abstract}
Resumen
La lengüeta o anquiloglosia, es una condición por la cual el frenillo lingual se adhiere cerca de la punta de la lengua y puede ser corto, apretado y grueso. La atadura de la lengua se ha citado como una causa de mala lactancia y dolor en los pezones maternos. La frenectomía, que se realiza comúnmente, puede corregir la restricción del movimiento de la lengua y permitir una lactancia más eficaz con menos dolor en el pezón materno. Tradicionalmente, la intervención quirúrgica se realiza con un bisturí, sin embargo en los últimos años ha habido un advenimiento importante del uso de láseres en este procedimiento. Objetivo: realizar una revisión sistemática sobre el uso de láseres como terapia para la anquiloglosia. Métodos: revisión sistemática de estudios clínicos aleatorizados con puntuación $\geq 6$ en la escala PEDro (Physiotherapy Evidence Database) publicados en las bases de datos que incluyen PubMed, Cochrane y SciELO. Se seleccionaron los estudios que evaluaron el uso del láser en el tratamiento de niños con anquiloglosia. Resultados: Se analizaron en total 7 estudios que utilizaron láser de Diodo o $\mathrm{CO}_{2}$ como una de las técnicas de frenectomía. En 401 pacientes sometidos a frenectomía lingual se describe claramente el beneficio entre realizar la técnica láser sobre la técnica tradicional del bisturí. Conclusión: La cirugía de frenectomía con láseres proporciona un tratamiento más eficiente y cómodo para el paciente infantil en comparación con los métodos tradicionales de bisturí / hoja. Sin embargo, no existe consenso ni evidencia que indique el tipo de láser a utilizar.
\end{abstract}

Palabras clave: Anquiloglosia; Frenectomía lingual; Aplicaciones láser.

\title{
1. Introdução
}

A anomalia oral congênita, conhecida como anquiloglossia caracterizada pelo freio lingual curto e anteriorizado, alterando os movimentos e funções da língua, como sucção, fala e alimentação (Junqueira et al., 2014), esta pode ser observada em recém-nascidos, crianças, bem como em adolescentes e adultos (Leal, 2010). A anquiloglossia em bebês também está relacionada à dificuldade em amamentar, engasgar ou vomitar alimentos, atraso no desenvolvimento ou deterioração da fala e de problemas comportamentais (Ghaheri et al., 2018).

A etiologia da anquiloglossia permanece desconhecida, entretanto parece estar associada a alguns fatores como sexo masculino e história familiar positiva. (Ballard et al., 2002) Embora seja um distúrbio relativamente comum, com prevalência variando de 0,1 a 10,7\%, o indivíduo afetado pode apresentar problemas relacionados à amamentação, mastigação, deglutição, fala, articulação e alterações dentárias.(Choi et al., 2011; Hong et al., 2010; Lalakea \& Messner, 2003; Puthussery et al., 2011) Diante das consequências da anquiloglossia, em muitas situações é necessário intervir e adaptar a mobilidade da língua e suas funções, o que resulta na melhoria da qualidade de vida do indivíduo. (Komori et al., 2017; Messner, Lalakea, 2002).

A frenectomia lingual é uma técnica de remoção total do freio lingual que se apresenta total ou parcialmente inserido no assoalho bucal (Puthussery et al., 2011). Esta cirurgia pode ser efetuada de modo convencional com lâmina de bisturi ou com recursos térmicos como o bisturi elétrico ou lasers (Aras et al., 2010; Gontijo; Navarro; Haypek; Ciamponi; Haddad, 2005; Hong et al., 2010; Navarro; Gontijo; Haypek; Eduardo, 2009).

\section{Metodologia}

Para a elaboração deste estudo descritivo foi realizada busca de periódicos indexados nas bases de dados: Web of Sciences, Medline e EMBASE por séries de casos, relatos de casos, estudos randomizados, revisões sistemáticas sobre o tema que apresentavam relatos de frenectomia por laser em pacientes pediátricos com anquiloglossia.

A questão que norteou esta revisão foi "Qual a eficácia do uso de lasers no tratamento de anquiloglossia em pacientes pediátricos?" Com base na formulação da questão norteadora, uma revisão de literatura sobre o uso de lasers como terapia para anquiloglossia (Higgins, Thomas, Chandler, Cumpston, Li, Page, 2019) As buscas foram realizadas nas bases de dados eletrônicas PUBMED, SciELO e Cochrane, escolhidas por sua ampla utilização nas ciências da saúde e por serem fontes de referência internacionalmente reconhecidas. 
Para a busca, foram utilizados os seguintes termos e combinações: Ankyloglossia x Laser; Frenectomia x Laser. Os termos de pesquisa estão listados em Medical Subjet Headings (MeSH) e Health Sciences Descriptors (DeCS). Apesar de anquiloglossia e frênulo lingual não serem mencionados, os autores optaram por mantê-los por serem termos comumente utilizados pela comunidade científica e pela prática clínica. As pesquisas foram delimitadas por idioma, inglês, português e espanhol, até a data de publicação, sendo definidas como recortadas de janeiro de 2009 a dezembro de 2019 e realizadas especificamente em seres humanos. Em casos de desacordo, um terceiro e quarto autores tomavam a decisão final.

\section{Seleção De Artigos}

A busca inicial no banco de dados eletrônico identificou 204 referências, 75 excluídas após limitar a data, estudos em humanos e omitindo duplicatas. Foram selecionados 129 artigos, sendo 52 excluídos com base no título e 42 excluídos com base no resumo. No total, 35 artigos foram submetidos à leitura na íntegra. Após os critérios de inclusão e exclusão, 7 artigos incluídos foram selecionados para a presente revisão conforme mostrado (Figura 1). (Baxter \& Hughes, 2018; Ghaheri et al., 2017, 2018; Gujrathi et al., 2016; Hanna \& Parker, 2016; Junqueira et al., 2014; Komori et al., 2017).

\section{Resultados e Discussão}

Tendo em vista relatos de mães, e pesquisadores, em estudos de levantamento biográficos, crianças com anquiloglossia que apresentam dificuldade de amamentação, problemas na pronúncia de letras, que podem ser originados devido às limitações de certos movimentos articulatórios, é indicada, procedimentos cirúrgicos de frenotomia imediata, ou o mais cedo possível, sendo cada vez mais comum sua realização em bebês recém-nascidos. A frenotomia com laser em bebês mostra-se uma técnica cirúrgica

Tem sido bem aceito que o laser cirúrgico é uma alternativa excelente e previsível aos métodos tradicionais de bisturi ou lâmina para frenectomias pediátricas. Alguns estudos (Baxter \& Hughes, 2018), descreveram pacientes com restrições posteriores da língua submetidos à frenectomia com laser de $\mathrm{CO}_{2}$ sem qualquer anestesia geral ou sedação. Após o procedimento, todos os pacientes demonstraram aumento da mobilidade lingual evidenciado pela melhora nas habilidades de fala e alimentação.

Tabela 1 - Artigos que corresponderam a busca.

\begin{tabular}{|c|c|c|c|c|}
\hline Autor & Desenho & Objetivo & Intervenção & Conclusões \\
\hline $\begin{array}{l}\text { Baxter et al., } \\
2018\end{array}$ & érie de casos & $\begin{array}{l}\text { Apresentar uma série de } \\
\text { cinco casos de pacientes } \\
\text { com restrição posterior da } \\
\text { língua, submetidos à } \\
\text { frenectomia com laser de } \\
\mathrm{CO}_{2} \text { sem qualquer } \\
\text { anestesia geral ou sedação }\end{array}$ & Laser de $\mathrm{CO}_{2}$ & $\begin{array}{l}\text { Os dados apresentados demonstram que mesmo os } \\
\text { ligamentos posteriores restringem o movimento e } \\
\text { afetam as estruturas orais que são necessárias para a } \\
\text { fala e a alimentação. Todos os pacientes demonstraram } \\
\text { aumento da mobilidade lingual evidenciado pela } \\
\text { melhora nas habilidades de fala e alimentação. Todos } \\
\text { os casos relatados foram frenectomias com laser de } \\
\mathrm{CO}_{2} \text {, que causam menos dor pós-procedimento do que } \\
\text { procedimentos com bisturis. O uso de laser também } \\
\text { permite excelente hemostasia e controle cirúrgico, bem } \\
\text { como notável cicatrização de feridas sem necessidade } \\
\text { de suturas. }\end{array}$ \\
\hline
\end{tabular}


Ghaheri et al., Estudo Coorte Determinar o impacto da 2017 Prospectivo frenectomia lingual $\mathrm{e}$ labial e comprometimento da amamentação

Ghaheri et al., Estudo Coorte 2018 Prospectivo

Determinar como a frenectomia lingual amarrado pode resultar em dificuldades persistentes na amamentação.

Avaliar e demonstrar as
Komori et al., Estudo clínico 2017

Junqueira et Série de casos
al., 2014

Hanna et al., Estudo Coorte 2016 Prospectivo

Investigar pacientes pediátricos com frênulo e a utilidade do tratamento com laser de $\mathrm{CO}_{2}$.

Descrever os casos abordados pelas diversas técnicas, informações sobre as indicações, contraindicações, vantagens e desvantagens. vantagens da aplicação do laser de $\mathrm{CO}_{2}$ em pacientes pediátricos.

Gujrathi et al., Clinical trial Avaliar a superioridade 2016 randomizado e do bipolar e do laser de
Laser Diodo.

Melhorias pós-operatórias significativas foram relatadas entre as pontuações pré-operatórias médias em comparação com as pontuações de $1 \mathrm{~s}$ e $1 \mathrm{~m}$ do BSES-SF, o I-GERQ-R e escala de dor VAS O consumo médio de leite materno melhorou $155 \%$ de 3,0 para $4,9 \mathrm{~mL} / \mathrm{min}$ ( $\mathrm{p}<0,001)$. As melhorias foram demonstradas em ambos os bebês com frênulo anterior clássico e ligadura posterior.

Melhorias pós-operatórias significativas foram relatadas entre os escores pré-operatórios médios em comparação com os escores de 1 semana e 1 mês da BSES-SF, F (2) = 41,2, p <0,001; o I-GERQ-R, F (2) = 22,7, p <0,001; e escala de dor VAS, F (2) = 46,1, p $<0,001$. Foi demonstrado que, além da dor nos mamilos, as medidas dos sintomas de refluxo do bebê e da autoconfiança na amamentação materna podem melhorar após a liberação total do frênulo lingual.

Laser de $\mathrm{CO}_{2}$. Demonstrou a utilidade do laser de $\mathrm{CO}_{2}$ na realização da frenectomia e ofereceu sugestões quanto ao momento desse procedimento.

Tesoura,

Todas as técnicas apresentadas tem sucesso no hemostasia e tratamento da anquiloglossia e requerem profissional Laser Diodo. habilitado. O laser pode ser considerado uma alternativa simples e segura para crianças, reduzindo a quantidade de anestésicos locais, o sangramento e as chances de infecção, edema e desconforto.

Laser de $\mathrm{CO}_{2}$.

O uso do laser tem demonstrado ajudar a reduzir o estresse e o medo da criança durante a cirurgia, o que pode ser considerado uma ferramenta essencial no manejo comportamental. A escolha do laser de $\mathrm{CO}_{2}$ é baseada na otimização de tais benefícios em relação às necessidades específicas de tratamento cirúrgico e de tecidos.

A: Bisturi. Os lasers de $\mathrm{CO}_{2}$ fornecem uma melhor percepção do B: Bipolar. paciente em termos de tempo operatório reduzido. O controlado $\quad \mathrm{CO}_{2}$ em relação à técnica

C: Laser. de bisturi convencional para frenectomia bipolar fornece uma melhor percepção do paciente em termos de dor pós-operatória e custo-benefício. Levando em consideração o admirável resultado clínico, tanto o eletro-cautério quanto o laser de $\mathrm{CO}_{2}$ oferecem uma alternativa segura, eficaz, aceitável e 
Legenda: M: Masculino F: Feminino; a: anos; m: meses, s: semanas. Fonte: Autores.

\section{Conclusões}

A frenectomia por cirurgia a laser oferece um tratamento mais eficiente e confortável para o paciente infantil em comparação aos métodos tradicionais de bisturi / lâmina. Em geral, os lasers atualmente utilizados em cirurgia bucal, como os lasers de $\mathrm{CO}_{2}$ e de Diodo, apresentam boas características e bons resultados em frenectomias. As evidências científicas revelam, principalmente por meio de relatos de experiência clínica, bons resultados com o uso dos dois tipos de lasers, no que diz respeito à capacidade de corte com eficiência e precisão, à coagulação intraoperatória. Em suma, as evidências científicas coletadas e analisadas não são suficientes para determinar diferenças nas características relacionadas à frenectomia entre os lasers de $\mathrm{CO}_{2}$ e Diodo, não sendo possível definir o tipo específico de laser para terapia proposta, levando-se em consideração alguma das características estudadas.

\section{Referências}

Aras, M. H., Göregen, M., Güngörmüş, M., \& Akgül, H. M. (2010). Comparison of diode laser and Er:YAG lasers in the treatment of Ankyloglossia. Photomedicine and Laser Surgery, 28(2), 173-177. https://doi.org/10.1089/pho.2009.2498

Ballard, J. L., Auer, C. E., \& Khoury, J. C. (2002). Ankyloglossia: assessment, incidence, and effect of frenuloplasty on the breastfeeding dyad. Pediatrics, 110(5). https://doi.org/10.1542/peds.110.5.e63

Baxter, R., \& Hughes, L. (2018). Speech and Feeding Improvements in Children After Posterior Tongue-Tie Release : A Case Series. 7(3), 29-35.

Choi, Y. S., Lim, J. S., Han, K. T., Lee, W. S., \& Kim, M. C. (2011). Ankyloglossia correction: Z-plasty combined with genioglossus myotomy. Journal of Craniofacial Surgery, 22(6), 2238-2240. https://doi.org/10.1097/SCS.0b013e3182320122

Ghaheri, B. A., Cole, M., Fausel, S. C., Chuop, M., \& Mace, J. C. (2017). Breastfeeding improvement following tongue-tie and lip-tie release: A prospective cohort study. Laryngoscope, 127(5), 1217-1223. https://doi.org/10.1002/lary.26306

Ghaheri, B. A., Cole, M., \& Mace, J. C. (2018). Revision Lingual Frenotomy Improves Patient-Reported Breastfeeding Outcomes: A Prospective Cohort Study. Journal of Human Lactation, 34(3), 566-574. https://doi.org/10.1177/0890334418775624

Gontijo, I., Navarro, R. S., Haypek, P., Ciamponi, A. L., \& Haddad, A. (2005). The Applications of diode and Er:Yag Lasers in labial frenectomy in infant patients. J Dentistry for Children., 72(1), 10-15.

Gujrathi, A. B., Ambulgekar, V., \& Handal, A. (2016). Surgical Techniques for the Treatment of Tongue Tie in Children : A Comparative Study. 16(1), 1-8. https://doi.org/10.9734/BJMMR/2016/24168

Hanna, R., \& Parker, S. (2016). The advantages of carbon dioxide laser applications in paediatric oral surgery . A prospective cohort study. Lasers in Medical Science. https://doi.org/10.1007/s10103-016-1978-8

Higgins, J. P. T., Thomas, J., Chandler, J., Cumpston, M., Li, T., Page, M. J. W. V. (editors). (2019). Cochrane Handbook for Systematic Reviews of Interventions. 2nd Edition. Chichester (UK), John Wiley, 1-23.

Hong, P., Lago, D., Seargeant, J., Pellman, L., Magit, A. E., \& Pransky, S. M. (2010). Defining ankyloglossia: A case series of anterior and posterior tongue ties. International Journal of Pediatric Otorhinolaryngology, 74(9), 1003-1006. https://doi.org/10.1016/j.ijporl.2010.05.025

Junqueira, M. A., Cunha, N. N. O., Costa e Silva, L. L., Araújo, L. B., Moreti, A. B. S., Couto filho, C. E. G., \& Sakai, V. T. (2014). Surgical techniques for the treatment of ankyloglossia in children: a case series. Journal of Applied Oral Science, 22(3), 241-248. https://doi.org/10.1590/1678-775720130629

Komori, S., Matsumoto, K., Matsuo, K., Suzuki, H., \& Komori, T. (2017). Clinical Study of Laser Treatment for Frenectomy of Pediatric Patients. International Journal of Clinical Pediatric Dentistry, 10(3), 272-277. https://doi.org/10.5005/jp-journals-10005-1449

Lalakea, M. L., \& Messner, A. H. (2003). Ankyloglossia: The adolescent and adult perspective. Otolaryngology - Head and Neck Surgery, 128(5), 746-752. https://doi.org/10.1016/S0194-5998(03)00258-4

Leal, R. A. (2010). Frenectomia Labiale Lingualem Odonto-pediatria. Monografia de Investigação/Relatório de Atividade Clínica. Universidade do Porto., Jun.

Messner A. H., Lalakea, M. L. (2002). The effect of ankyloglossia on speech in children. Otolaryngology - Head and Neck Surgery, 127(5), 452-454. https://doi.org/10.1067/mhn.2002.129823

Navarro, R. S., Gontijo, I., Haypek, P., \& Eduardo, C. (2009). Lasers em odontopediatria: estado da arte. Odontopediatria na primeira infância. 
Research, Society and Development, v.10, n. 2, e19210210434, 2021

(CC BY 4.0) | ISSN 2525-3409 | DOI: http://dx.doi.org/10.33448/rsd-v10i2.10434

Puthussery, F. J., Shekar, K., Gulati, A., \& Downie, I. P. (2011). Use of carbon dioxide laser in lingual frenectomy. British Journal of Oral and Maxillofacial Surgery, 49(7), 580-581. https://doi.org/10.1016/j.bjoms.2010.07.010 